\title{
Z-scan measurement with an astigmatic Gaussian beam
}

\author{
Yong-Liang Huang and Chi-Kuang Sun \\ Department of Electrical Engineering and Graduate Institute of Electro-Optical Engineering, \\ National Taiwan University, Taipei 10617, Taiwan
}

Received March 26, 1999

Z-scan measurement with a slit instead of a circular aperture is proposed and analyzed for an astigmatic Gaussian beam. This new technique can accurately determine not only the nonlinear refractive index but also the ellipticity and positions of $x-y$ foci of the astigmatic beam. (c) 2000 Optical Society of America [S0740-3224(99)02311-5]

OCIS codes: 190.3270, 190.5940, 190.7110, 120.4570.

\section{INTRODUCTION}

Z-scan is a popular technique for measurement of nonlinear refractive index $\mathrm{n}_{2}{ }^{1}$ because of its simplicity and accuracy. A circular symmetric Gaussian beam is used in the measurement. This method was described in detail by Sheik-Bahae et al. ${ }^{1}$ Briefly, a single symmetric Gaussian beam is focused by a lens. A nonlinear material moves through the beam focus in the beam propagation direction (called the $z$ direction). As a result of either self-focusing or self-defocusing, the variation of the far-field transmittance through a finite circular aperture is then measured. From the shape and the peak-tovalley difference of the Z-scan trace, the sign as well as the magnitude of $n_{2}$ can be obtained.

However, an astigmatic Gaussian beam such as a modelocked laser beam, a frequency-doubled laser beam, or a semiconductor laser beam, is often encountered in the laboratory. Astigmatic Gaussian beams have different spot sizes and different beam-waist positions in the two directions (called the $x$ and $y$ directions) orthogonal to the beam-propagation direction. The effects of beam ellipticity on Z-scan measurement were previously studied by Mian et al. ${ }^{2}$ Their study showed that a Z-scan trace is greatly influenced by ellipticity and beam-waist separation. The peak-to-valley difference in the Z-scan trace was found to decrease nonlinearly as a function of ellipticity. Assumption of a circular Gaussian beam regardless of its ellipticity will produce a great deviation in Z-scan signatures. This means that a circular aperture is no longer suitable for Z-scan measurement in this situation. In this paper we propose a new Z-scan measurement for which we use a slit instead of a circular aperture. With a numerical simulation, we show that accurate $n_{2}$, ellipticity, and focus positions of the astigmatic Gaussian beam can all be obtained with this new technique.

\section{THEORY}

Z-scan measurement with elliptical Gaussian beams were previously studied by Mian et al. ${ }^{2}$ The far-field pattern of the Gaussian beam at the aperture plane was derived by a Gaussian decomposition method. ${ }^{1}$ The complex electric field at the exit plane of the sample was decomposed into a summation of Gaussian beams through a Taylor series expansion of the nonlinear phase term. Each Gaussian beam could simply propagate to the aperture plane, where the single beams would be recombined. The far field at the aperture plane was described by Eq. (19) of Ref. 2 and is rewritten here in the following form:

$$
\begin{aligned}
& E_{a}(x, y, z, t) \\
&=E(0,0, z, t) \exp (-\alpha L / 2) \\
& \times \sum_{m=0}^{\infty}\left[\frac{w_{m 0 x}(z) w_{m 0 y}(z)}{w_{m x}(z) w_{m y}(z)}\right]^{1 / 2} \exp \left[i \eta_{m}(z)\right] \\
& \times \exp \left[-\frac{i k x^{2}}{2 q_{m x}(z)}-\frac{i k y^{2}}{2 q_{m y}(z)}\right] \frac{\left.i \Delta \phi_{0}(z, t)\right]^{m}}{m !},
\end{aligned}
$$

where $\mathrm{L}$ is thickness of the nonlinear material, $\alpha$ is the linear absorption coefficient, and $\mathrm{w}_{\mathrm{mox}} / \mathrm{w}_{\mathrm{m} 0 \mathrm{y}} / \mathrm{w}_{\mathrm{mx}} / \mathrm{w}_{\mathrm{my}}$, $\eta_{\mathrm{m}}$, and $\mathrm{q}_{\mathrm{mx}} / \mathrm{q}_{\mathrm{my}}$, respectively, are parameters related to the waist, phase, and q parameter of the Gaussian beam at the aperture plane. ${ }^{2} \quad \mathrm{n}_{2}$ is related to nonlinear phase changes $\Delta \Phi_{0}$ as $\Delta \Phi_{0}=\mathrm{n}_{2} \mathrm{~kL}$ eff $2 \mathrm{P}(\mathrm{t}) / \pi \mathrm{w}_{0 \mathrm{x}} \mathrm{w}_{0 \mathrm{y}}$, where $\mathrm{k}$ is the wave number and the effective thickness is $L_{\text {eff }}=(1$ $\left.-\mathrm{e}^{-\alpha \mathrm{L}}\right) / \alpha$. $\mathrm{L}$ should be small enough that changes in the beam diameter within the sample caused by either diffraction or nonlinear refraction can be neglected. ${ }^{1} P(t)$ is the input power. $w_{0 x}$ and $w_{0 y}$ are the beam waists located at focus positions $z_{x}$ and $z_{y}$, respectively.

The far-field transmittance after the slit for cubic nonlinearity can be derived from Eq. (1). When a slit perpendicular to the y direction, called the y slit, is inserted to measure the field intensity, the on-axis normalized transmission after the slit that is due to nonlinear phase change $\Delta \Phi_{0}$ is given by

$$
T_{y}(z)=\frac{\int_{-y_{0} / 2}^{y_{0} / 2} \int_{-\infty}^{\infty}\left|E_{a}\left(x, y, z, \Delta \Phi_{0}\right)\right|^{2} d x d y}{\int_{-y_{0} / 2}^{y_{0} / 2} \int_{-\infty}^{\infty}\left|E_{a}(x, y, z, 0)\right|^{2} d x d y},
$$


where $\mathrm{y}_{0}$ is the slit width in the $y$ direction and the slit is centered in the y direction. Because the y slit is an open aperture in the $x$ direction, the self-focusing or selfdefocusing effect in the $x$ direction will not be measured. The transmittance is labeled $\mathrm{T}_{\mathrm{y}}$, indicating that only variations of transmittance in the $y$ direction are measured. Z-scan trace $T_{x}$ can be derived in a similar way, which measures the variation of transmittance in the $x$ direction. For a small nonlinear phase change $\left(\Delta \Phi_{0}\right.$ $<0.3$ ), only the first three summation terms in Eq. (1) should be considered.

\section{DISCUSSION}

\section{A. Elliptical Gaussian Beam}

If an astigmatic Gaussian beam has the same $x-y$ foci, the Z-scan traces measured by a slit will be similar to those measured by a circular aperture. The only difference between the traces is in the magnitude of differences between peak and valley. Figure 1(a) shows Z-scan traces measured by an $x$ slit and a y slit for focused beam radii of $\mathrm{w}_{0 \mathrm{x}}=20 \mu \mathrm{m}$ and $\mathrm{w}_{0 \mathrm{y}}=10 \mu \mathrm{m}$ with a small nonlinearity $\Delta \Phi_{0}=0.1$. A Z-scan trace measured by a small circular aperture is also shown in Fig. 1(a), labeled $\mathrm{T}_{\mathrm{c}}$, for comparison. These three traces are all symmetric. Trace $T_{y}$ measures the variation in the $y$ direction where the smaller beam radius is located and trace $T_{x}$ is related to the larger beam radius in the $x$ direction; the variation of trace $T_{y}$ is larger than that of trace $T_{x}$. Similarly, trace $T_{c}$ measures the variation in both the $x$ direction and the $y$ direction, so the variation of trace $T_{c}$ is the largest. Because we can obtain separate information from the $x$ and the $y$ slits, the ellipticity of the elliptical Gaussian beam can be obtained from the peak-to-valley difference ratio of traces $T_{x}$ and $T_{y}$. We select the larger variation trace, $T_{y}$, to calculate $n_{2}$.

As the ellipticity increases, the variation of the Z-scan trace that corresponds to the smaller focused beam radius becomes larger, and that which corresponds to the larger focused beam radius becomes smaller. For example, if the beam radii are $\mathrm{w}_{0 \mathrm{x}}=40 \mu \mathrm{m}$ and $\mathrm{w}_{0 \mathrm{y}}=10 \mu \mathrm{m}$, the corresponding Z-scan traces are as shown in Fig. 1(b). Now the variation of trace $T_{y}$ is larger than that of trace $T_{c}$.

The Z-scan trace is measured through a finite aperture, so the peak-to-valley difference in the Z-scan trace is influenced by the aperture size, which is defined by linear transmittance S. ${ }^{1}$ For measurement with a slit, similar to the case with a circular aperture, a larger slit width corresponds to a larger linear transmittance, which corresponds to a smaller peak-to-valley difference in the measured Z-scan trace.

Because the $Z$-scan traces $T_{x}$ and $T_{y}$ are related to the beam radi in the $x$ and $y$ directions, respectively, information on ellipticity can be obtained from these traces. Ellipticity $\xi$ can thus be described as a function of the $x-y$ peak-to-valley difference ratio $\left(\Delta \mathrm{T}_{\mathrm{y}} / \Delta \mathrm{T}_{\mathrm{x}}\right)$ and the linear transmittance through slit $\mathrm{S}$, as shown in Fig. 2. A fitting equation to this nonlinear relation is

$$
\xi=\left(0.398+0.662 x-0.0375 x^{2}\right) S^{-k},
$$

where

$$
\mathrm{x}=\frac{\Delta \mathrm{T}_{\mathrm{y}}}{\Delta \mathrm{T}_{\mathrm{x}}}, \quad \mathrm{k}=-0.0167+\frac{0.319}{4(\mathrm{~S}-0.0972)^{2}+0.0162}
$$

For an ellipticity larger than 3, the peak-to-valley difference of trace $T_{x}$ becomes small and the peak-to-valley difference ratio $\Delta \mathrm{T}_{\mathrm{y}} / \Delta \mathrm{T}_{\mathrm{x}}$ becomes large. The approximate equation is no longer accurate. However, for a highly elliptical beam an accurate value of ellipticity is not needed for determination of the nonlinear refractive index, as we show in Subsection 3.B.

Because the peaks and valleys of a Z-scan trace are the result of a nonlinear phase change, nonlinear $\mathrm{n}_{2}$ can be obtained from the peak-to-valley difference of a Z-scan trace. $^{1}$ We obtained two Z-scan traces, $T_{x}$ and $T_{y}$, in two
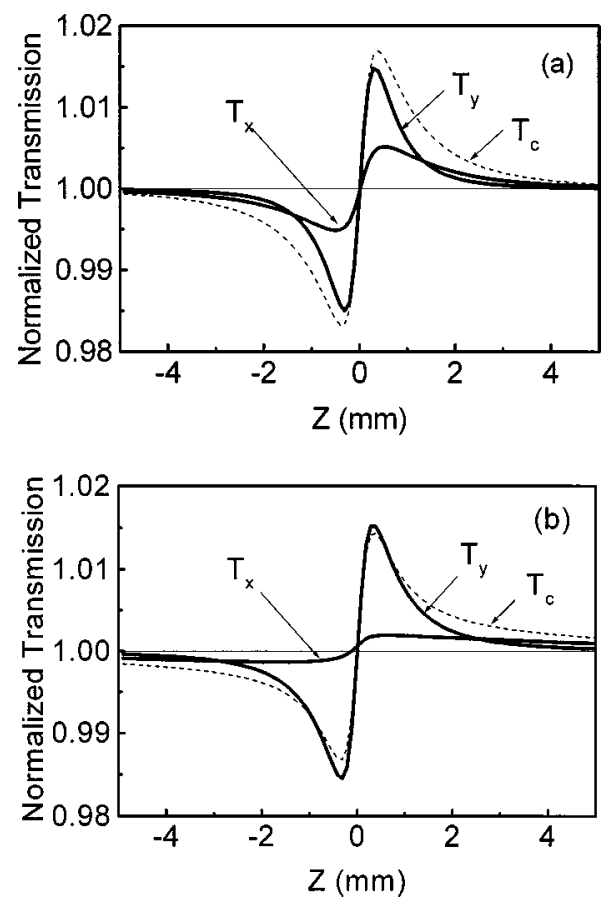

Fig. 1. (a) Z-scan traces with focused beam radii of $w_{0 x}$ $=20 \mu \mathrm{m}$ and $\mathrm{w}_{0 \mathrm{y}}=10 \mu \mathrm{m}$. Traces $\mathrm{T}_{\mathrm{x}}$ and $\mathrm{T}_{\mathrm{y}}$ were measured with slits perpendicular to the $x$ and $y$ directions, respectively. Trace $T_{c}$ was measured with a circular aperture. (b) Z-scan traces with focused beam radii of $w_{0 x}=40 \mu \mathrm{m}$ and $w_{0 y}$ $=10 \mu \mathrm{m}$.

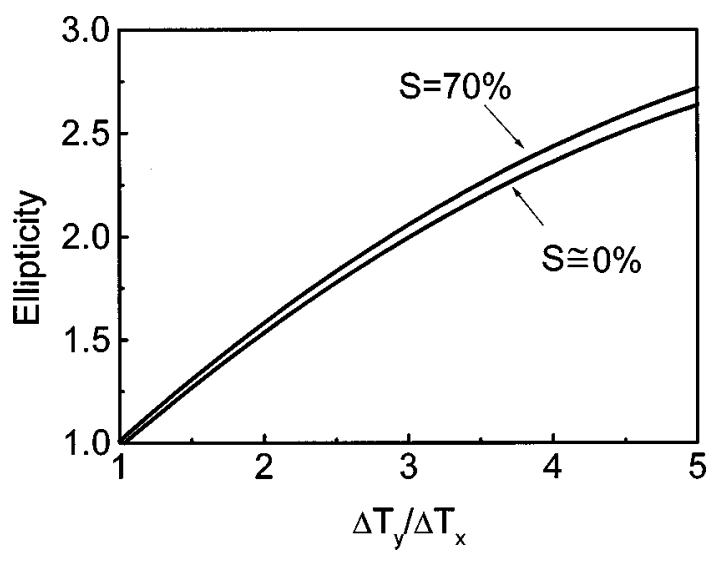

Fig. 2. Ellipticity versus peak-to-valley difference ratio. 


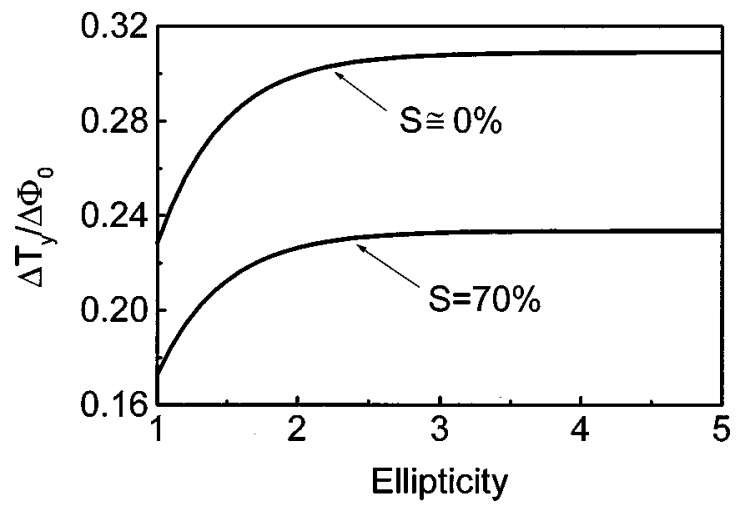

Fig. 3. Ratio between dominant peak-to-valley difference and nonlinear phase change versus ellipticity.

orthogonal directions. We selected the dominant trace to calculate the nonlinear $n_{2}$. $T_{y}$ was taken as the dominant trace in our examples. The ratio between nonlinear phase change $\Delta \Phi_{0}$ and peak-to-valley difference $\Delta T_{y}$ versus ellipticity is shown in Fig. 3, which is also found to be a function of ellipticity and slit linear transmittance. A fitting equation to this nonlinear relation can be given as follows:

$$
\frac{\Delta \mathrm{T}_{\mathrm{y}}}{\Delta \Phi_{0}}=\left\{0.309-0.0804 \exp \left[-\frac{(\xi-1)}{0.472}\right]\right\} \mathrm{S}^{-\mathrm{k}},
$$

where

$$
k=-0.119+\frac{0.259}{4(S-0.957)^{2}+0.0234} .
$$

If $\mathrm{S}<10 \%$, the term $\mathrm{S}^{-\mathrm{k}}=1$ should be used in Eq. (5). For an elliptical Gaussian beam with $\xi<3$, the ellipticity of the elliptical Gaussian beam can be obtained from Eq. (3). When the ellipticity is larger than 3 , the $\Delta T_{y} / \Delta \Phi_{0}$ ratio is almost independent of ellipticity and is only a function of slit linear transmittance. Nonlinear phase change $\Delta \Phi_{0}$ is then obtained from $\Delta T_{\mathrm{y}} / \Delta \Phi_{0}$ $\cong 0.309 \mathrm{~S}^{-\mathrm{k}}$. Nonlinear $\mathrm{n}_{2}$ is related to the nonlinear phase change by $\Delta \Phi_{0}=\mathrm{n}_{2} \mathrm{~kL}$ eff $2 \mathrm{P}(\mathrm{t}) / \pi \mathrm{w}_{0 \mathrm{x}} \mathrm{W}_{0 \mathrm{y}}$, and nonlinear $\mathrm{n}_{2}$ can thus be accurately determined.

\section{B. Astigmatic Gaussian Beam}

Astigmatic Gaussian beams have different spot sizes and different beam-waist positions in two directions orthogonal to the beam-propagation direction. Z-scan traces were found to be greatly influenced by ellipticity and beam-waist separation. ${ }^{2}$ Figure 4 shows the Z-scan traces for a Gaussian beam with beam radii of $\mathrm{w}_{0 \mathrm{x}}$ $=40 \mu \mathrm{m}$ and $\mathrm{w}_{0 \mathrm{y}}=10 \mu \mathrm{m}$ located at $\mathrm{z}_{\mathrm{x}}=2000 \mu \mathrm{m}$ and $\mathrm{z}_{\mathrm{y}}=0 \mu \mathrm{m}$, respectively. As the nonlinear material moves close the foci, the smaller beam radius $w_{0 y}$ $=10 \mu \mathrm{m}$ is encountered first, and the dominant Z-scan trace $T_{y}$ is observed. Trace $T_{x}$ corresponds to the larger beam radius $\mathrm{w}_{0 \mathrm{x}}=40 \mu \mathrm{m}$ located at $\mathrm{z}_{\mathrm{x}}=2000 \mu \mathrm{m}$.

Because the power intensity is higher in the neighborhood of the smaller beam radius, the nonlinear phase change in this region is thus larger. The valley of trace $T_{x}$ is located in this region, and it is greatly enhanced by this effect. It becomes large, and the peak vanishes. If the ellipticity of a Gaussian beam is large ( $>3$, the influ- ence of the larger beam radius on dominant trace $T_{y}$ is negligible, and trace $T_{y}$ is almost perfectly symmetric. This indicates that dominant trace $T_{y}$ is a suitable $Z$-scan trace for measurement with an astigmatic Gaussian beam with large ellipticity $(>3)$. On the other hand, the previous method with a circular aperture will yield a trace $T_{c}$ that measures the variation in both $x$ and the $y$ directions. Because of the contribution from the $x$ component, trace $T_{c}$ becomes seriously asymmetric, and its valley is much larger than its peak. Previous studies ${ }^{1,2}$ showed that nonlinear absorption can also suppress the peak and enhance the valley of a Z-scan trace, and thus an asymmetric Z-scan trace can be produced. ${ }^{1}$ Asymmetric Z-scan trace $T_{c}$ induced by beam-waist separation is thus similar to that caused by nonlinear absorption, and it is hard to distinguish the difference. Fortunately, with our proposed new technique this ambiguity does not occur to dominant trace $T_{y}$ because trace $T_{y}$ is al most symmetric without nonlinear absorption.

If the ellipticity of a Gaussian beam is small $(<3)$, the role of the larger beam radius becomes increasingly more important as the ellipticity decreases. Figure 5 shows the Z-scan traces for a Gaussian beam with radii of $w_{0 x}$ $=20 \mu \mathrm{m}$ and $\mathrm{w}_{0 \mathrm{y}}=10 \mu \mathrm{m}$ located at $\mathrm{z}_{\mathrm{x}}=2000 \mu \mathrm{m}$ and $z_{y}=0 \mu \mathrm{m}$, respectively. The peak of dominant trace $T_{y}$ is located in the region between two foci and is slightly enhanced by the high power intensity in this region. On the other hand, the valley of trace $T_{x}$ is greatly enhanced

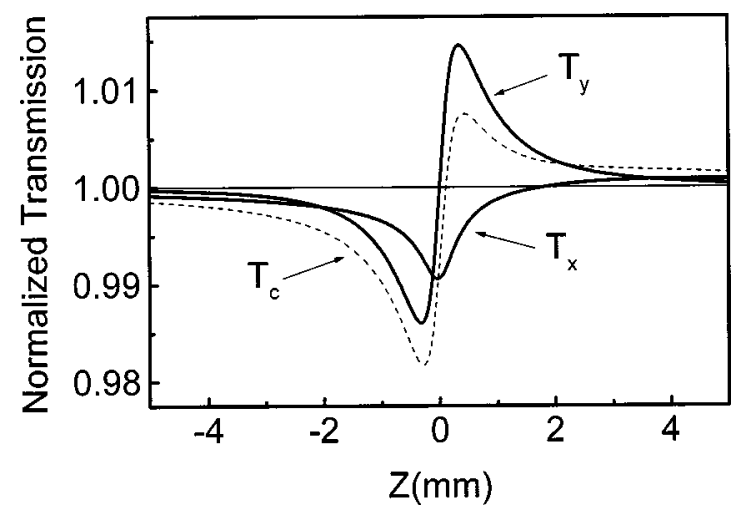

Fig. 4. Z-scan traces with focused beam radii of $\mathrm{w}_{0 \mathrm{x}}=40 \mu \mathrm{m}$ and $\mathrm{w}_{0 \mathrm{y}}=10 \mu \mathrm{m}$ located at $\mathrm{z}_{\mathrm{x}}=2000 \mu \mathrm{m}$ and $\mathrm{z}_{\mathrm{y}}=0 \mu \mathrm{m}$, respectively.

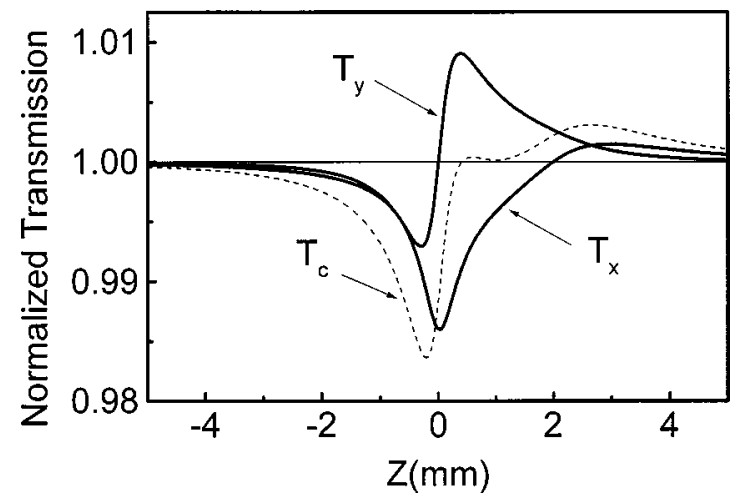

Fig. 5. Z-scan traces with focused beam radii of $\mathrm{w}_{0 \mathrm{x}}=20 \mu \mathrm{m}$ and $\mathrm{w}_{0 \mathrm{y}}=10 \mu \mathrm{m}$ located at $\mathrm{z}_{\mathrm{x}}=2000 \mu \mathrm{m}$ and $\mathrm{z}_{\mathrm{y}}=0 \mu \mathrm{m}$, respectively. 
by the high power intensity near the smaller beam radius. Compared with that obtained by the conventional circular aperture technique, trace $T_{c}$ is distorted and contains double peaks. Even though trace $T_{y}$ does show slight asymmetry for an astigmatic Gaussian beam with small ellipticity $(<3)$, dominant trace $\mathrm{T}_{\mathrm{y}}$ still is more advantageous than the conventionally obtained trace $T_{c}$.

Z-scan traces were also found to be influenced by beamwaist separation. A large beam-waist separation not only increases the distortion of Z-scan traces but also decreases the peak-to-valley difference of the dominant trace. Figure 6 shows Z-scan traces for a Gaussian beam with beam radii of $\mathrm{w}_{0 \mathrm{x}}=20 \mu \mathrm{m}$ and $\mathrm{w}_{0 \mathrm{y}}=10 \mu \mathrm{m}$ located at $z_{x}=1000 \mu \mathrm{m}$ and $z_{y}=0 \mu \mathrm{m}$, respectively. Compared with Z-scan trace $T_{y}$ shown in Fig. 5, trace $T_{y}$ in Fig. 6 is more symmetric, and the peak-to-valley difference is larger. Although trace $T_{C}$ becomes less distorted when the beam-waist separation is smaller it is still confused with a Z-scan trace with nonlinear absorption.

Under the conditions discussed above, the smaller beam radius was encountered first. If the larger beam is encountered first, the shapes of the Z-scan traces are different. Figure 7 shows Z-scan traces for a Gaussian beam with beam radii of $\mathrm{w}_{0 \mathrm{x}}=40 \mu \mathrm{m}$ and $\mathrm{w}_{0 \mathrm{y}}=10 \mu \mathrm{m}$ located at $z_{x}=2000 \mu \mathrm{m}$ and $z_{y}=0 \mu \mathrm{m}$, respectively. The larger beam radius is encountered first in this situation. The peak of trace $T_{x}$ is greatly enhanced by the higher power intensity between the two foci. Dominant trace $T_{y}$ in measurements with slits remains symmetric and unchanged because it is highly elliptical. For comparison, in Fig. 7 we also show $T_{c}$, which is asymmetric because of astigmatism. All these examples indicate that dominant trace $T_{y}$ in measurements with slits is much more symmetric than trace $T_{c}$ in measurements with circular apertures. This result is attributed to the decoupling between information from the $x$ and the $y$ axes in our proposed measurements. This shape symmetry makes the Z-scan measurements more accurate and the following analysis less confusing.

Z-scan traces $T_{x}$ and $T_{y}$ can also provide information on beam-waist positions. The point at which dominant trace $T_{y}$ crosses 1 is exactly the smaller beam-waist position. For a Gaussian beam with small ellipticity $(<2)$, the crossing point of trace $T_{x}$ and 1 is exactly the larger beam-waist position. However, as the ellipticity increases, the variation of trace $T_{x}$ becomes smaller, and it is easily influenced by the smaller beam radius in the $y$ direction. The crossing point of trace $T_{x}$ with 1 thus shows a little difference from the larger beam-waist position. For example, $5 \%$ of the deviation is found for an ellipticity of 3. With increased ellipticity, the deviation also increases.

In Subsection 3.A we discussed how to obtain the ellipticity of an elliptical Gaussian beam with the same $x-y$ beam-waist positions. Although Z-scan traces become asymmetric (especially for $T_{x}$ ) because of the effect of beam-waist separation, the ellipticity still can be determined by traces $T_{x}$ and $T_{y}$. If the beam-waist separation is small $(<200 \mu \mathrm{m})$, the shape of the Z-scan trace is not distorted greatly, and the ellipticity calculated by Eq. (3) is still valid. With a beam-waist separation of $75 \mu \mathrm{m}$, the deviation is only $1 \%$. As the beam-waist separation

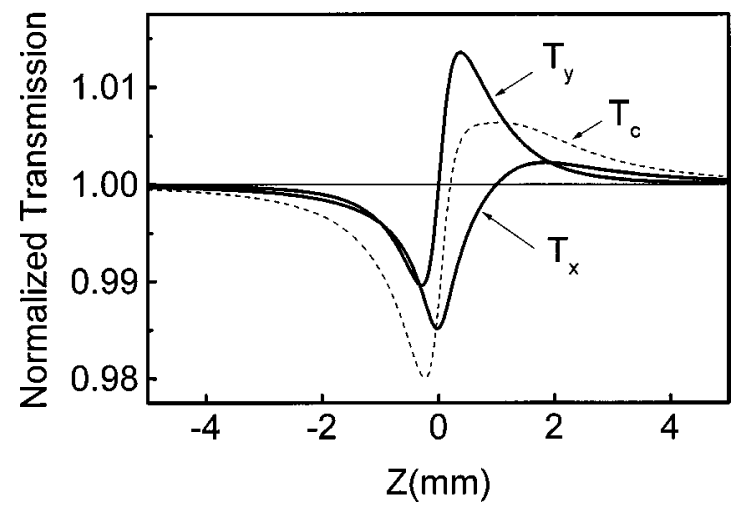

Fig. 6. Z-scan traces with focused beam radii of $\mathrm{w}_{0 \mathrm{x}}=20 \mu \mathrm{m}$ and $w_{0 y}=10 \mu \mathrm{m}$ located at $z_{x}=1000 \mu \mathrm{m}$ and $z_{y}=0 \mu \mathrm{m}$, re spectively.

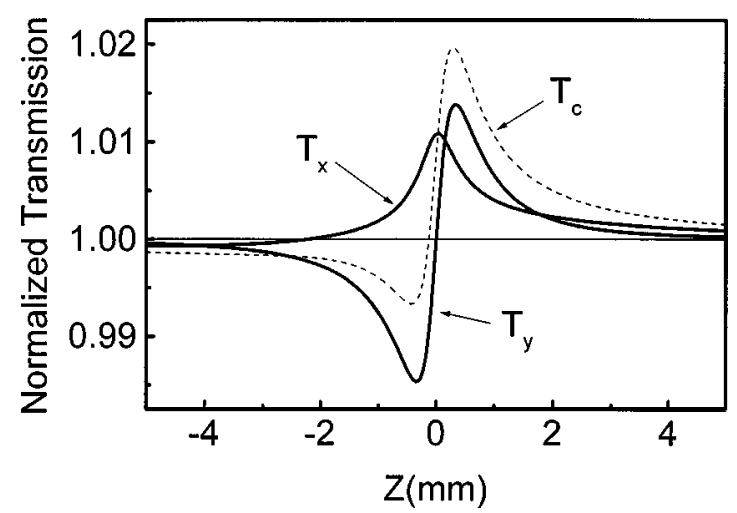

Fig. 7. Z-scan traces with focused beam radii of $\mathrm{w}_{0 \mathrm{x}}=40 \mu \mathrm{m}$ and $w_{0 y}=10 \mu \mathrm{m}$ located at $z_{x}=2000 \mu \mathrm{m}$ and $z_{y}=0 \mu \mathrm{m}$, re spectively.

increases, Z-scan traces become increasingly more distorted. The deviation is less than $8 \%$ for a beam-waist separation of $200 \mu \mathrm{m}$. If the beam-waist separation is even larger, the ellipticity should be obtained by another.

In Subsection 3.A we also discussed the determination of the value of the nonlinear $n_{2}$ by dominant trace $T_{y}$ for an elliptical Gaussian beam with the same beam-waist positions. Although Z-scan traces are influenced by beam-waist separation, dominant trace $T_{y}$ is still quite symmetric. This indicates that accurate $\mathrm{n}_{2}$ can still be determined by the dominant trace with measurements that use a slit instead of a circular aperture. If the beamwaist separation is less than some specific value $\Delta_{x y}$, Eq. (5) is still found to be highly accurate. For a deviation of less than $1 \%, \Delta_{x y}$ is a function of smaller beam radius $\mathrm{w}_{0 \mathrm{y}}$ and ellipticity $\xi$ and is given by

$$
\Delta_{x y}=w_{0 y}{ }^{2} f(\xi) \text {, }
$$

where

$$
f(\xi)=1.231-0.594 \xi+0.494 \xi^{2}
$$

which is obtained by fitting. As the beam-waist separation is greater than this range, the deviation increases.

\section{Two-Photon Absorption}

When two-photon absorption is considered in the $Z$ scan, it contributes to an additional nonlinear phase change. ${ }^{1}$ 
This results in a valley near the focus in the openaperture Z-scan trace. ${ }^{1,2}$ For the finite-aperture Z-scan trace, two-photon absorption would suppress the peak and enhance the valley. ${ }^{1}$ The properties of Z-scan traces measured by a slit with an astigmatic Gaussian beam are similar to those measured by a circular aperture with a circular Gaussian beam. The peak of the Z-scan trace is found to be suppressed and the valley is found to be enhanced. Figure 8 shows the Z-scan traces with the parameters of Fig. 4 with two-photon absorption considered. All the peaks of these traces are suppressed, and all the valleys are enhanced. When we compare trace $T_{c}$ in Fig. 4 with that in Fig. 8, the two traces are quite similar, and one may misunderstand that these two traces are all influenced by two-photon absorption. But trace $\mathrm{T}_{c}$ in Fig. 4 is induced by beam-waist separation and trace $\mathrm{T}_{c}$ in Fig. 8 is caused by both beam-waist separation and twophoton absorption. On the other hand, when a slit is

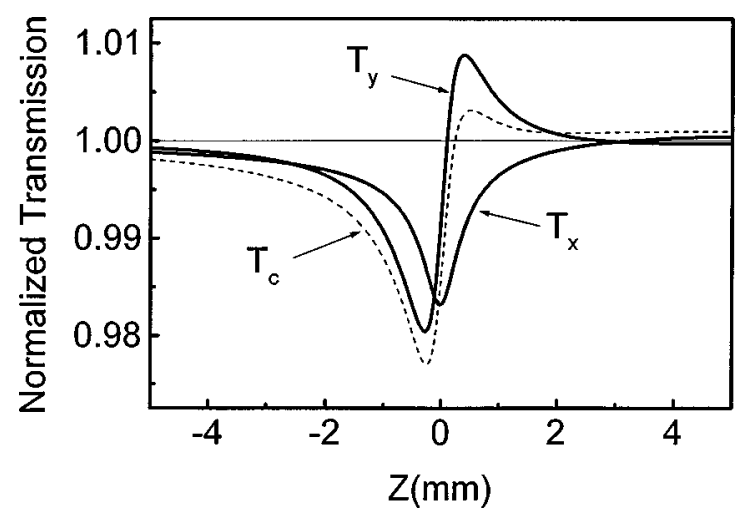

Fig. 8. Z-scan traces with two-photon absorption considered for focused beam radii of $\mathrm{w}_{0 \mathrm{x}}=40 \mu \mathrm{m}$ and $\mathrm{w}_{0 \mathrm{y}}=10 \mu \mathrm{m}$ located at $\mathrm{z}_{\mathrm{x}}=2000 \mu \mathrm{m}$ and $\mathrm{z}_{\mathrm{y}}=0 \mu \mathrm{m}$, respectively. used in Z-scan measurement, dominant trace $T_{y}$ has an advantage in this situation. It is almost symmetric without two-photon absorption, even for astigmatic Gaussian beam. The variation is apparent with two-photon absorption.

\section{SUMMARY}

In summary, we have proposed a Z-scan measurement by using a slit instead of a circular aperture. By measuring Z-scan traces and using the slit in two orthogonal directions, we obtained accurate values of the nonlinear refractive index, ellipticity, and foci of an astigmatic Gaussian. This new technique has advantages compared with the conventional technique, especially for an astigmatic Gaussian beam with large beam-waist separation and high ellipticity.

\section{ACKNOWLEDGMENT}

This project is supported by National Science Council of Taiwan under contracts NSC 88-2112-M-002-003 and NSC 88-2218-E-002-038.

C.-K. Sun's e-mail address is sun@cc.ee.ntu.edu.tw.

\section{REFERENCES}

1. M. Sheik-Bahae, A. A. Said, T.-H. Wei, D. J . Hagan, and E. W. V. Stryland, "Sensitive measurement of optical nonlinearities using a single beam," IEEE J. Quantum Electron. 26, 760-769 (1990).

2. S. M. Mian, B. Taheri, and J. P. Wicksted, "Effect of beam ellipticity on Z-scan measurements," J . Opt. Soc. Am. B 13, 856-863 (1996). 